\title{
Editorial
}

\section{Promoting exclusive breastfeeding in India to reduce neonatal mortality}

Neonatal period or the first 28 days after birth, is the most vulnerable time in a child's life. In the late 1990s almost half of infant deaths, in the first year of life, occurred in the neonatal period. As infant mortality declines, the proportion contributed by neonatal deaths tends to increase. With better prenatal, intrapartum and neonatal care, globally the neonatal mortality has reduced by almost $47 \%$ from 38 to 19 deaths per 1000 live births in the years 1990 and 2015, respectively. ${ }^{1}$ Neonatal mortality in India is, however, higher at 27.7 per 1000 live births in 2015. Hence there is an urgent need to identify and address risk factors for this high neonatal mortality in India.

Breastfeeding is known to improve infant survival and there are global efforts to promote it. However, systematic follow-up studies from India on effect of breastfeeding on neonatal survival are scarce. To fill in this lacuna, Srivastava and $\mathrm{Awasthi}^{2}$ have enrolled 1020 newborns delivered in urban health facilities and followed up 91.8\% during the neonatal period. Majority of the mothers (74.3\%) has taken adequate antenatal care and hence had opportunity to interact with health care providers and be informed about healthy neonatal care practices, including breastfeeding. Based on their feeding patterns the neonates were classified as exclusively breast fed, predominantly breast fed, when they received other non-milk fluids fed, and partially breast fed when they received top milk as well as other non-milk fluids. They report the occurrence of neonatal illnesses and found that exclusive breastfeeding was protective when compared to predominantly and partially breastfed infants, in models controlling for gestation age, single birth as well as birth weight. The authors did not study neonatal mortality as an endpoint. They have also not reported the factors facilitating breastfeeding. Large-scale prospective studies are needed in different states of India to identify barriers to exclusive breastfeeding and to revisit, critically evaluate and modify programs promoting exclusive breastfeeding to ensure their effectiveness.

In a study conducted in Ethiopia, exclusive breastfeeding was found to be the strongest predictor of infant survival. ${ }^{3}$ In Indian urban slum and non-slum population where the study was conducted, ${ }^{2}$ only $26.5 \%$ (248/937) neonates were exclusively breastfed. These figures are much lower than the $46.3 \%$ and $48.6 \%$ mothers exclusively breast feeding as reported in the National Family and Health Survey (NFHS)-1 and NFHS-3. ${ }^{4}$ The data reported by Srivastava and Awasthi ${ }^{2}$ seem more reliable as it was collected on prospective follow-up in contrast to NFHS data collected by recall.

In a meta-analysis of 3 studies from 5 publications between 1963 and 2011, it has been found that early (within $24 \mathrm{~h}$ of birth) initiation of breastfeeding improved neonatal outcomes like illnesses and survival among all live births as well as low birth weight babies. ${ }^{5}$ This meta-analysis did not include the study by Srivastava and Awasthi ${ }^{2}$ where they have carefully analyzed the data to exclude the effects of reverse causality, that is neonatal illness resulting in delayed initiation or non-initiation of breastfeeding, by only including those babies who were successfully discharged from the hospital and were breastfed. They reported that the partially breast bed babies had 2.5 times increased risk of mortality/ hospitalization as compared to exclusively breast fed babies. There is, therefore, an urgent need to improve breast-feeding practices in India to achieve the Sustainable Developmental Goals.

\section{REFERENCES}

1. Wardlaw T, You D, Hug L, Amouzou A, Newby H. UNICEF Report: enormous progress in child survival but greater focus on newborns urgently needed. Reprod Health. 2014;11:82.

2. Srivastava NM, Awasthi S. Breastfeeding pattern and the risk of neonatal illnesses among urban poor in Lucknow, Northern India: a prospective follow-up study. Clin Epidemiol Glob Health. 2016;4:153-162.

3. Biks GA, Berhane Y, Worku A, Gete YK. Exclusive breast feeding is the strongest predictor of infant survival in Northwest Ethiopia: a longitudinal study. J Health Popul Nutr. 2015;34:9.

4. Chandhiok N, Singh KJ, Sahu D, Singh L, Pandey A. Changes in exclusive breastfeeding practices and its determinants in India, 1992-2006: analysis of national survey data. Int Breastfeed J. 2015;10:34.

5. Debes AK, Kohli A, Walker N, Edmond K, Mullany LC. Time to initiation of breastfeeding and neonatal mortality and morbidity: a systematic review. BMC Public Health. 2013;13 (suppl 3):S19. http://dx.doi.org/10.1186/1471-2458-13-S3-S19. 
Avivar Awasthi Junior Resident, Department of Medicine, Kasturba Medical College, Manipal, Karnataka, India

Shally Awasthi* Professor, Department of Pediatrics, King George's Medical University, Lucknow, U.P., India
${ }^{*}$ Corresponding author E-mail address: shally07@gmail.com (S. Awasthi) http://dx.doi.org/10.1016/j.cegh.2016.11.001 2213-3984/ Published by Elsevier, a division of RELX India, Pvt. Ltd on behalf of INDIACLEN. 